\title{
VERIFICATION OF AFFORDANCE EFFECT OF HMI IN THE VR ENVIRONMENT
}

\author{
Maki Arame ${ }^{1,2}$, Junko Handa1, ${ }^{1,}$, Yoshiko Goda ${ }^{2}$, Masashi Toda ${ }^{2}$, Ryuichi Matsuba ${ }^{2}$, \\ \& Tatsuru Daimon ${ }^{3}$ \\ ${ }^{1}$ Polytechnic University of Japan, Tokyo (Japan) \\ ${ }^{2}$ Kumamoto University, Kumamoto-city (Japan) \\ ${ }^{3}$ Keio University, Tokyo (Japan)
}

\begin{abstract}
There are always big issues to use new technology. The users need to know how to use it, and what will happen if they don't use it properly because of lack of knowledge. Level 3 and 4 automated vehicles will be on the road shortly in Japan. Drivers and pedestrians need to deal with new types of cars to keep themselves safe. It is thought that there are individual differences in the perception of affordances when encountering self-driving cars. In this research, the environment of encounter with an automated vehicle were developed in VR that verified the difference in affordance perceptions depending on individual attributes. As a result, it was suggested that a metaphorical HMI of automated vehicle may promote proactive behavior.
\end{abstract}

Keywords: Automated vehicle, affordance, VR environment, person centered approach.

\section{Introduction}

Self-driving cars will soon be widespread on public roads. It is important not only the technological development of hardware and software that support automated driving but also the communication between automated vehicles and humans from the perspective of safety (Zhou, Itoh, \& Kitazaki, 2018; Zhou, Itoh, \& Kitazaki, 2019). This study investigated pedestrians' behaviors at a crosswalk when they encountered an automated vehicle. Considering their risks, an environment of the intersection in which pedestrians encountered an automated driving vehicle was created using Virtual Reality (VR).

This study investigated the factors that contributed to pedestrians' judgments that they could safely cross the crosswalk and their relation to personal attributes and external Human-Machine Interfaces(eHMI).

\section{Purpose of study}

A traffic safety education course for the general public has only about 5 minutes of teaching time. In order to prevent accidents caused by human error, Keskine (1996) states hierarchical approach theory of driving behavior consists of five levels. The first level includes basic driving skills and knowledge of laws and regulations, the second level includes a response to traffic others and hazard prediction. The third level includes skills related to the purpose of driving and the formulation of operation plans (setting travel times and routes). The fourth level is self-control skills such as awareness and responsibility to drive safely and avoid accidents. The fifth level encompasses social environment. Current traffic safety education mainly targets up to the second level, but in order to achieve safety education in the whole society, it is important for people to have a higher level of safety awareness (Keskinen, 2014).

In previous studies, it was confirmed that the learning effect may differ depending on individual attributes when the same content is taught in different teaching materials: pamphlet format, quiz format, and video format. The purpose of this study is to construct a walking environment with automated vehicles in a VR environment, and to examine the factors that increase safety awareness through the experience of participants encountering automated vehicles as pedestrians. 
Figure 1. Position of a pedestrian in a VR environment.

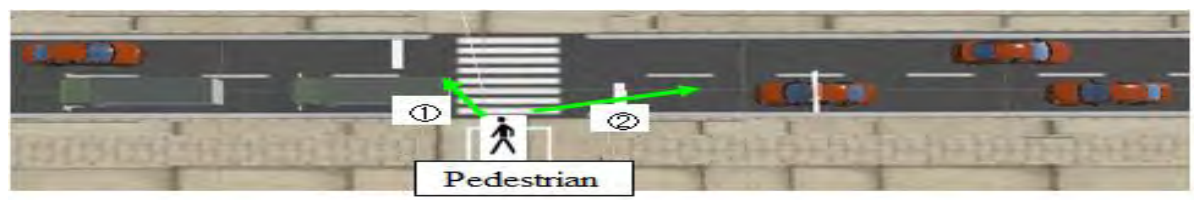

\section{Literature Review}

\subsection{Situation awareness model}

Endsley (2013) proposed a model of situational awareness, in which he states that people are required to accurately recognize situations as they dynamically change, to predict future situations, and to do what people can in the present to achieve a good situation in the future. In this process, the following three steps of situational awareness are key.

Level 1: Perceive the current situation surrounding you. Level 2: Understand the implications of the situation you notice. Level 3: Predict how the situation will unfold in the future. Then, decide what steps to take in the present to deal with the predicted future situation, and solemnly execute the decision. In the case of an encounter with an automated car, it notices the approach of an oncoming car (Level 1), understands what it means for its own path (Level 2), and predicts the future situation (Level 3). The situational awareness model points out the need to check the effectiveness of the support at each level.

To put it in the context of this study, it is whether the subject was able to recognize whether there was an automatic vehicle in the surroundings when walking (Level 1), whether the subject was able to understand the contents of the automatic vehicle's eHMI when recognizing the vehicle (Level 2), and whether the predictive development of the future situation change was presented (Level 3). If the situational awareness is appropriate, the time required to take coping actions is expected to be shorter.

\subsection{Experiential Learning Cycle}

Kolb proposes the Kolb's Experiential Learning Cycle. Kolb described four stages for learning abstract concepts that can be applied in the real world. The four stages are concrete experience, reflective observation, abstract conceptualization, and active experimentation (Kolb, 1984).

Concrete experience is when the learner has a new experience or encounters a new situation. Reflective observation is when the learner observes others or makes observations about his/her own experiences. Abstract conceptualization is the process of understanding what has happened and interpreting events and the relationships between them. Active experience is the learner's application of his or her ideas and concepts to the real world.

The experiential learning model recommends appropriate feedback during learning. The framework of feedback is divided into information notification and rebuilding. In the informational notification type of feedback, missing knowledge and information are compensated. For inexperienced people, it is to inform them of the situation and the results. In the rebuilding type of feedback, it is to support their self-reflection and to help them create action plans for improvement.

Feedback appears to be something that is pinpointed, but pedagogically, it is closely related to overall instructional design and goals. If appropriate feedback is provided during experiential learning with VR, it is thought that safety awareness when walking may be improved.

\section{Research Question}

The research questions were the following three points.

1. Do the messages make a difference in the pedestrian's behavior? If so, which message was appropriate for the pedestrian to initiate the crossing?

2. What messages cause pedestrians to observe their surroundings more carefully?

3. What kind of message will raise awareness of safety considerations for the driver and others?

\section{Method}

\subsection{Participants and procedure}

The survey data used in this study were a secondary analysis of the results of $101 \mathrm{VR}$ experiment data in 2020. In the survey, in addition to age and gender, driver's license existence was surveyed as basic attributes of respondents. A VR experiment was conducted in about 5 minutes, and when an automated 
vehicle equipped with eHMI approached a no-signal pedestrian crossing, one of three types of language messages ((1) after you, (2) I will stop, and (3) no message) was displayed.

In this study, the time from when the automatic vehicle stops until the subject starts crossing was measured. Furthermore, a questionnaire and an interview were conducted after the experiment to investigate the relationship between behavior and recognition by the displayed message. The experiment was conducted 11 times for one subject, and the data of the 11th time were analyzed in this study.

\subsection{Measurement}

In this study, the following three types of questionnaires are conducted after the VR crossing experiment. Answer each question in 5 stages (1: I do not agree at all-5: I strongly agree).

Q1. The first car approaching from the right side gave way to you.

Q2. I was able to cross with confidence before the first car approaching from the right side passed.

Q3. I tried to cross quickly, paying attention to the car that stopped.

After the experiment was completed, subjects were interviewed about "How did you think the driver would judge and act when the driver of the oncoming vehicle saw the situation of the self-driving car (including the contents if there was an outward HMI)?"

\section{Results}

\subsection{Results of pre-posttests by learning material type}

Table 1 summarizes the age and the presence or absence of a driver's license as individual attributes of the subjects for each message displayed in the experiment. The ages were classified into 12 to 15 years old (hereinafter, junior high school), 16 to 65 years old (hereinafter, non-elderly), and 65 years old and over (hereinafter, elderly). It is considered that there is a difference in judgment depending on whether the person has a driver's license or not.

Table 1. Subject attributes.

\begin{tabular}{l|c|c|r|r}
\hline eHMI & $\begin{array}{c}\text { elderly } \\
\text { (license holders) }\end{array}$ & $\begin{array}{l}16 \text { to 64 years } \\
\text { (license holders) }\end{array}$ & $\begin{array}{c}12 \text { to } 15 \text { years } \\
\text { (license holders) }\end{array}$ & $\begin{array}{l}\text { Total } \\
\text { (license holders) }\end{array}$ \\
\hline After you & $4(4)$ & $20(10)$ & $10(0)$ & $34(14)$ \\
\hline I will stop & $3(3)$ & $18(10)$ & $8(0)$ & $29(13)$ \\
\hline No message & $9(9)$ & $19(10)$ & $10(0)$ & $38(19)$ \\
\hline
\end{tabular}

\subsection{Results of coping time by pattern}

Figure 2 compared the average time from when the automated vehicle stopped to when it started crossing for each displayed message. A positive value of the coping time means the start of walking after the automated vehicle stops. And a negative value means the start of walking before the automated vehicle stops. It can be seen it takes less time to deal with "(1) after you" and "(2)I will stop" compared to the case "no message." In addition, since there was a significant difference in the time required for coping with each type of eHMI, it can be considered that the message influenced their judgement. By age groups, it can be seen the older the person, the longer the coping time, regardless of the type of message.

Figure 2. Comparison of coping time by age group by message.

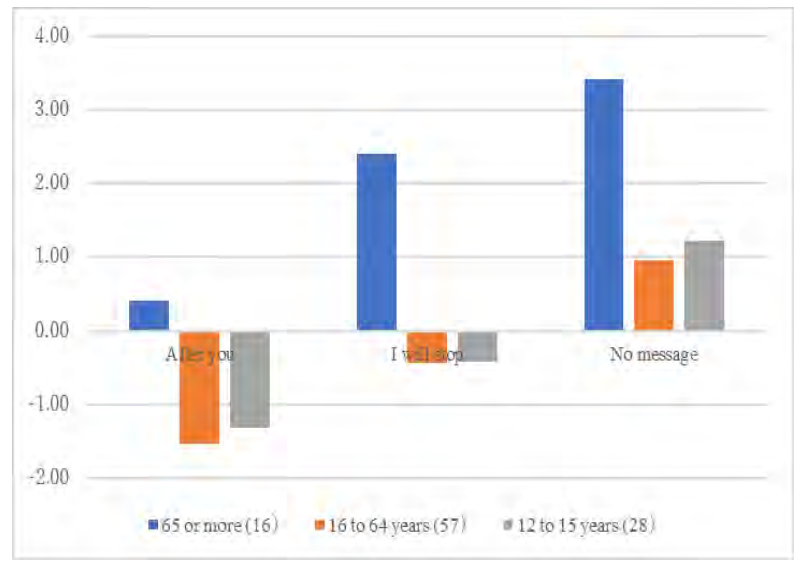




\subsection{Questionnaire analysis results}

Table 2 compared the average answers to each question for each message. The average answer for Q1 "The first car approaching from the right side gave way to you" was the highest "no message". And the average answer for Q2 "Did you cross with confidence?" had become "(1) after you" the lowest, then "(3) no message”, and "(2) I will stop." Q3 "I tried to cross quickly with care" had the highest average response "no message."

Although the presence of eHMI did not necessarily foster a sense of security, it has been confirmed that it may have the effect of predicting the next behavior.

Table 2. Average score for questions by message.

\begin{tabular}{l|c|r|r|r}
\hline eHMI & & Q1 & Q2 & Q3 \\
\hline \multirow{2}{*}{$\begin{array}{l}\text { After you } \\
\mathrm{N}=34\end{array}$} & Mean & 4.50 & 3.65 & 3.21 \\
\cline { 2 - 5 } I will stop & SD & 0.66 & 1.37 & 1.25 \\
$\mathrm{~N}=29$ & Mean & 4.52 & 4.10 & 3.00 \\
\hline \multirow{2}{*}{$\begin{array}{l}\text { No message } \\
\mathrm{N}=38\end{array}$} & SD & 0.74 & 0.98 & 1.41 \\
\cline { 2 - 5 } & Mean & 4.55 & 3.89 & 3.24 \\
\hline
\end{tabular}

Table 3 compared the average answers to each question for each message for car license holders. It was considered that the driver's license holders made a judgment based on their driving experiences so far. As result of the analysis, it can be seen the average score of Q1 and Q2 is the highest and the average score of Q3 is the lowest for "I will stop". This result shows that "I will stop" was the most appropriate eHMI for license holders.

Table 3. Average answers by question for car license holders.

\begin{tabular}{|c|c|c|c|c|}
\hline eHMI & & Q1 & Q2 & Q3 \\
\hline \multirow{2}{*}{$\begin{array}{l}\text { After you } \\
\mathrm{N}=14\end{array}$} & Mean & 4.36 & 3.43 & 3.00 \\
\hline & SD & 0.74 & 1.34 & 1.24 \\
\hline \multirow{2}{*}{$\begin{array}{l}\text { I will stop } \\
\mathrm{N}=13\end{array}$} & Mean & 4.46 & 4.00 & 2.92 \\
\hline & SD & 0.78 & 1.22 & 1.19 \\
\hline \multirow{2}{*}{$\begin{array}{l}\text { No message } \\
\mathrm{N}=19\end{array}$} & Mean & 4.42 & 3.79 & 3.11 \\
\hline & SD & 0.90 & 1.23 & 0.99 \\
\hline
\end{tabular}

Figure 3 shows a comparison of the average scores of Q1, Q2, and Q3 between license holders and non-license holders. It can be seen that the average of license holders was low in Q1 and Q2 regardless of the message, and the average of license holders was high in Q3. "After You" showed that the difference between licensed and unlicensed was greater than other eHMI.

Figure 3. Score comparison by questionnaire by message with or without driver's license.

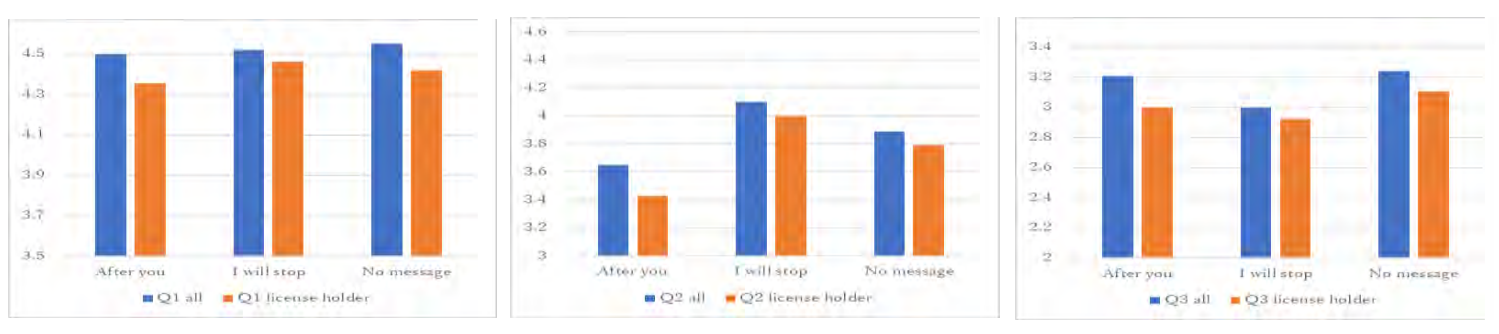

\subsection{Free description analysis results}

Table 4 was an analysis of the interview, "How did you think the driver would judge and act when the driver of the oncoming vehicle looked at the situation of the self-driving car (including the contents if there was an outward HMI)?" The results of morphological analysis using text analysis software (KH Coder ver3) were as follows. In the chi-square test of independence, there were significant differences in three items. It can be seen the subjects of "after you" highly evaluate HMI as a judgment material, while "I will stop" had a high evaluation other than HMI. There was no significant difference in the morphological analysis results whether the person was the license holder or not. From this, learning of automated vehicle by VR may have had a greater impact than previous driving experience. 
Table 4. Results of morphological analysis.

\begin{tabular}{l|r|r|r|r|r} 
& \multicolumn{1}{|l|}{ Not stop } & \multicolumn{1}{l|}{ Slow down } & \multicolumn{1}{l|}{ Stop } & unknown & look at the HMI \\
\hline after you & $2(5.88 \%)$ & $19(55.88 \%)$ & $26(76.47 \%)$ & $2(5.88 \%)$ & $11(32.35 \%)$ \\
\hline I will stop & $0(0.00 \%)$ & $12(41.38 \%)$ & $18(62.07 \%)$ & $1(3.45 \%)$ & $4(13.79 \%)$ \\
\hline No message & $7(20.00 \%)$ & $7(20.00 \%)$ & $24(68.57 \%)$ & $3(8.57 \%)$ & $0(0.00 \%)$ \\
\hline Chi-square value & $8.29 *$ & $9.47 * *$ & 1.55 & $\begin{array}{r}0.73 \\
* \mathrm{p}<0.5 * * \mathrm{p}<0.01\end{array}$
\end{tabular}

\section{Conclusions}

In this study, we examined the effect of eHMI on learning with the aim of introducing a VR environment into traffic safety education for pedestrians. Based on the situational awareness model, we investigated the coping time and recognition for each eHMI, and confirmed that the response time and consideration contents differed depending on the eHMI. Especially a message of "After you" made a pedestrian hurry. Thus, it is less time to start to walk. Yet, they may not have observed surroundings that may be a risk for them. Instead, "I will stop" made a pedestrian feel safe although it takes a time to start crossing. "No message" took a long time for a pedestrian to start crossing, but they check their surroundings. Pedestrians who have driver's licenses seemed to be more careful to initiate crossing. In conclusion, there were differences in the pedestrian's behavior depending on the messages. A message seemed to influence pedestrians' judgment. It may be based on their personal experience. In this study, which message raised awareness of safety were not able to discover, they need to have experience for automated driving vehicles.

\section{Limitations and recommendations}

In this VR experiment, detailed education on automated vehicles was not given before the experiment. In the future, after experiencing VR learning materials, we will make a plan to consider the implications of implementing explicit guidance and feedback methods. The eHMI of this study was compared only with three types, "after you", "I will stop", and no message. In the future, it will be necessary to verify whether similar trends can be seen in more diverse HMIs.

\section{Acknowledgments}

This work was supported by Council for Science, Technology and Innovation (CSTI), Cross-ministerial Strategic Innovation Promotion Program (SIP), entitled "Human Factors and HMI Research for Automated Driving" (funding agency: NEDO).

\section{References}

Endsley, M. R. (2013). Situation awareness. In J. D. Lee \& A. Kirlik (Eds.), Oxford library of psychology. The Oxford handbook of cognitive engineering (p. 88-108). Oxford University Press.

Keskinen, E. (1996). Why do young drivers have more accidents? Junge Fahrer und Fahrerinnen. Referate der Esten Interdiziplinären Fachkonferenz 12-14. Dezember 1994 in Köln. Berichte der Bundesanstalt für Strassenwesen. Mensch und Sicherheit, Heft M 52.

Keskinen, E. (2014). Education for older drivers in the future. IATSS Research, Vol38, Issue1, pp. 14-21.

Kolb, D. A. (1984). Experiential Learning: Experience as the Source of Learning and Development. Englewood Cliffs, NJ: Prentice Hall.

Zhou, H., Itoh, M., Kitazaki S. (2018). How Does Knowledge about System Limitations Contribute to Interventions into Partial Automation Among Elderly Drivers? Proceedings of the 2018 IEEE international Conference on Systems, Man, and Cybernetics (SMC2018), pp. 851-820.Miyazaki, Japan,

Zhou, H., Itoh, M., Kitazaki, S. (2019). Long-term Effect of Experiencing System Malfunction on Driver Take-over Control in Conditional Driving Automation. Proceedings of the 2019 IEEE international Conference on Systems, Man, and Cybernetics (SMC2019), pp. 1950-1955. Bari, Italy (2019). 